\title{
Progress in protecting vestibular hair cells
}

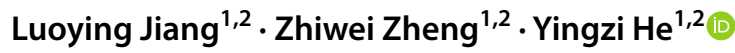

Received: 17 March 2021 / Accepted: 29 April 2021 / Published online: 13 May 2021

(c) The Author(s) 2021

\begin{abstract}
Vestibular hair cells are mechanosensory receptors that are capable of detecting changes in head position and thereby allow animals to maintain their posture and coordinate their movement. Vestibular hair cells are susceptible to ototoxic drugs, aging, and genetic factors that can lead to permanent vestibular dysfunction. Vestibular dysfunction mainly results from the injury of hair cells, which are located in the vestibular sensory epithelium. This review summarizes the mechanisms of different factors causing vestibular hair cell damage and therapeutic strategies to protect vestibular hair cells.
\end{abstract}

Keywords Vestibular dysfunction · Hair cells · Ototoxic drugs · Protection

\section{Introduction}

Peripheral vestibular organs are composed of two otolith organs: a utricle and a saccule, and three semicircular canals with different orientations: anterior, posterior, and horizontal semicircular canals, where vestibular hair cells (HCs) are mechanosensory receptors that are capable of detecting the position changes of the head so that animals can adjust their posture and coordinate their movement (Merchant et al. 2000). Healthy HCs are therefore essential for performing vestibular physiological functions. Among the population, vestibular dysfunction is prevalent, and it increases remarkably with age (Agrawal et al. 2009). In the US, about $18 \%$ of the population aged $40-49$ years, and $49 \%$ of the population aged 60-69 years suffer from vestibular dysfunction. The morbidity of this disorder arrives at $80 \%$ among people over 80 years old in the US (Negishi-Oshino et al. 2019).

Vestibular HCs are vulnerable to gene factors, aging, and exposure to therapeutic drugs, such as aminoglycoside and cisplatin. Extensive loss of vestibular sensory cells can cause

Luoying Jiang and Zhiwei Zheng contributed equally to this work.

Yingzi He

yingzihe09611@126.com

1 ENT Institute and Department of Otorhinolaryngology, Eye and ENT Hospital, State Key Laboratory of Medical Neurobiology and MOE Frontiers Center for Brain Science, Fudan University, Shanghai 200031, China

2 NHC Key Laboratory of Hearing Medicine, Fudan University, Shanghai 200031, China dysfunction of the peripheral vestibular apparatus, eliciting multiple unpleasant symptoms, such as imbalance, instability, dizziness, and vertigo (Burns and Stone 2017). With symptoms getting more severe, patients are likely to become depressed due to a general decline in their physical functions (Brosel and Strupp 2019). These symptoms may exert a negative impact on one's quality of life and increase the health care expenditure of a society.

However, research on the inner ear is mainly focused on the cochlea, while the damage factors and protective strategies of vestibular HCs have received relatively little attention. Furthermore, there are currently no equivalent therapeutic strategies for vestibular dysfunction compared to some effective hearing loss treatments such as cochlear implants and hearing aid devices (Wall et al. 2002). Therefore, how to protect vestibular HCs from loss and injury is an important issue.

\section{Structural and functional features of vestibular hair cells}

The sensory epithelium of the otolith organ, called macula, detects linear or translational acceleration, and the sensory epithelium of the semicircular canals, called the crista ampullaris, senses angular acceleration (Kingma and van de Berg 2016). The macula is composed of the otolithic membrane and the macular epithelium (Jeong et al. 2020). The otolithic membrane consists of a gelatinous membrane and some otoliths consisting of calcium carbonate (Kingma and van de 
Berg 2016) and the macular epithelium comprises sensory HCs and supporting cells (Jeong et al. 2020) (Fig. 1). The histological morphology of the crista ampullaris is similar to the macula, while its gelatinous membrane called cupula has no otoliths, and is thicker than that of the macula (Kingma and van de Berg 2016) (Fig. 2).

Vestibular HCs are primary motion sensors in the vestibular sensory epithelium (Kingma and van de Berg 2016). A vestibular $\mathrm{HC}$ consists of a cell body and a bundle of cilia on their apical end, which includes a kinocilium, and about 50 stereocilia (Hudspeth and Corey 1977). The kinocilium is the longest cilium, and the closer the stereocilia are to the kinocilia, the longer the stereocilia are. At the top of the cilia is a mechanical connection called elastic tip links, enabling the cilia of one hair cell to move together (Kingma and van de Berg 2016). The striola is a curve for the relative alignment of stereocilia in the macula. The cilia of HCs in the utricle are oriented toward the striola, while cilia in the saccule are oriented away from the striola (Khan and Chang 2013) (Fig. 3). Two types of HCs, Type I hair cells and Type II hair cells, differ in morphology (Ji and Zhai 2018). Type I hair cells have a goblet shape whose bases are surrounded by afferent nerve fibers. Type II hair cells have a columnar shape and the bottom of the cell body connects with the synapses of afferent nerve fibers (Khan and Chang 2013) (Fig. 1).

In response to the stimulation of linear or angular acceleration, the stereociliary bundle of one $\mathrm{HC}$ tilts toward the kinocilium, which leads to the activation of the mechanosensitive channel at the tips of the cilia. This can lead to the absorption of $\mathrm{K}^{+}$through top channels of the cilia, depolarizing the membrane of HCs. This depolarization can cause calcium influx by opening voltage-dependent calcium channels at the bases of HCs, stimulating the release of neurotransmitters to increase the firing frequency of nerve fibers (Negishi-Oshino et al. 2019).

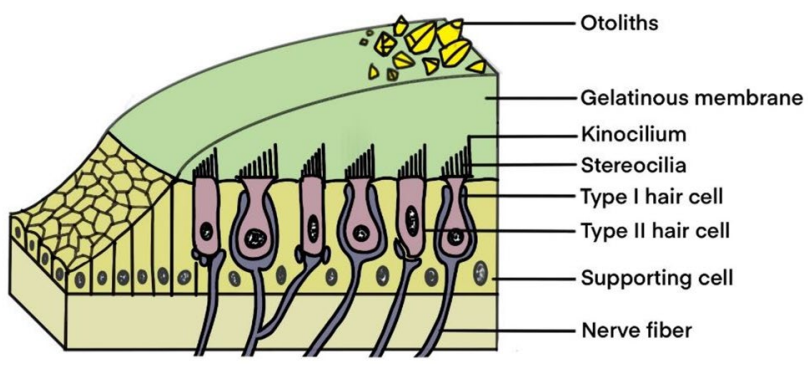

Fig. 1 Structure of the macula. The macula is composed of the otolithic membrane and macular epithelium. The otolithic membrane consists of a gelatinous membrane and some otoliths. The macular epithelium comprises sensory hair cells and supporting cells

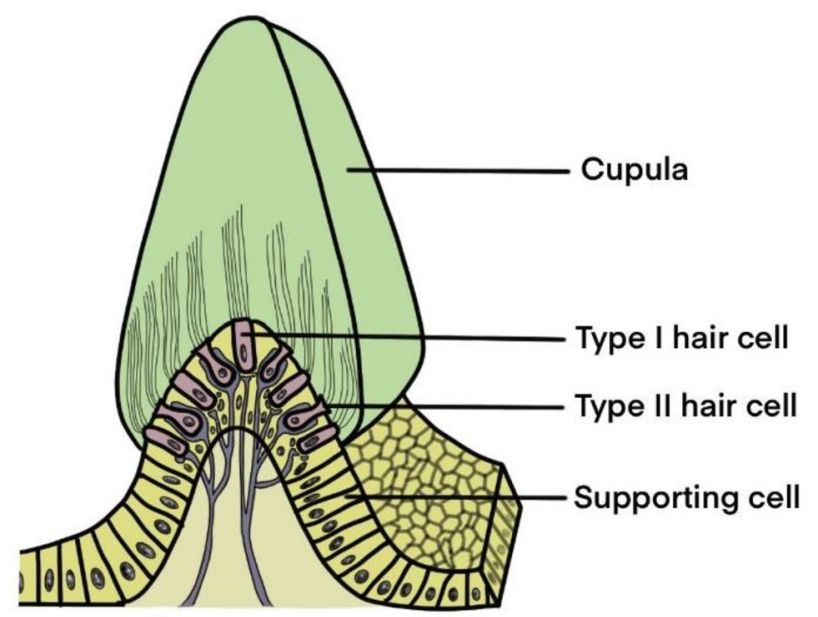

Fig. 2 Structure of the crista ampullaris. The histological morphology of the crista ampullaris is similar to the macula, while its gelatinous membrane called the cupula has no otoliths and is thicker than that of the macula

\section{Pathology and mechanisms of vestibular hair cell injury}

Generally speaking, human vestibular dysfunction can be attributed to the injury or loss of vestibular HCs. These cells are hypersensitive to certain therapeutic drugs, aging, and genetic factors. Vestibulotoxicity is characterized by the damage or loss of HCs in the utricle, saccule, or semicircular canals.

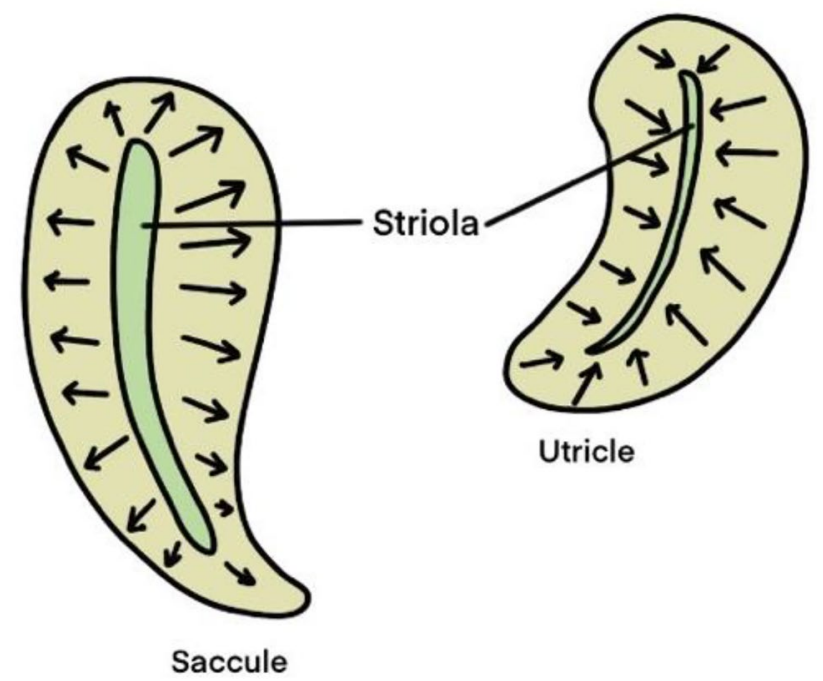

Fig. 3 Structure of the striola in saccule and utricle. Cilia of hair cells in the saccule are oriented away from the striola, while cilia of hair cells in the utricle are oriented toward the striola 


\section{Drugs}

The main drugs that cause vestibular ototoxicity are anticancer drug cisplatin and aminoglycoside antibiotics.

\section{Cisplatin}

Cisplatin is a widely used antineoplastic agent (Wu et al. 2017), which has toxic effects on nerves, kidneys, and sensory epithelia in the vestibular and cochlear system (Ding et al. 2018), causing vestibular dysfunction as well as hearing loss. Although cochlear injury of cisplatin has been widely studied, the mechanisms of cisplatin-induced vestibulotoxicity have been relatively less reported (Wu et al. 2017).

It has been reported that cisplatin not only markedly reduced the density of HCs in the utricle, saccule, and ampullae but also gave rise to abnormal morphology and disorganization of vestibular HCs (Tian et al. 2013; Wu et al. 2017; Zhang et al. 2003). Hair cells in the extra-striolar region are more susceptible to cisplatin than those in the striolar area (Cunningham and Brandon 2006). Many studies have suggested that cisplatin-induced vestibular HC death presumably mainly occurs by apoptosis (Kim et al. 2008; Wu et al. 2017; Zhang et al. 2003).

Some studies have noted that cisplatin causes dosedependent $\mathrm{HC}$ loss in the utricle. The number of HCs decreased with the dose of cisplatin increased from 5 to $10 \mu \mathrm{g} / \mathrm{mL}$ (Zhang et al. 2003), 0 to $25 \mu \mathrm{g} / \mathrm{mL}$ (Cunningham and Brandon 2006), or from 0 to $50 \mu \mathrm{g} / \mathrm{mL}$ (Lentz et al. 2013). However, Ding et al. reported that the dose-response of vestibular HCs to cisplatin was U-shaped rather than linear. They treated the utricle, saccule, and ampullae of rats with $10-1000 \mu \mathrm{M}$ cisplatin. When the concentration of cisplatin increased from 10 to $50 \mu \mathrm{M}$, the number of vestibular HCs decreased. Conversely, when the dose of cisplatin increased from 100 to $1000 \mu \mathrm{M}$, the number of vestibular HCs began to increase. Interestingly, when treated with $1000 \mu \mathrm{M}$ of cisplatin, almost HCs in cultures were survival, and the maximum HC loss occurred about 50-100 $\mu \mathrm{M}$ (Ding et al. 2018). The author suggested that this might because high levels of cisplatin disrupt the uptake of substances at the top vestibular HCs (Ding et al. 2018). The different dose-response curves of vestibular HCs exposure to cisplatin may be due to the application of a wider concentration range of cisplatin in Ding's experiment, reflecting the effect of higher doses of cisplatin on vestibular HCs that had not been reflected before.

It has been clarified that the cytotoxicity of cisplatin is associated with the impairment of DNA (Leibbrandt et al. 1995; Ries and Klastersky 1986) and mitochondria (Sugiyama et al. 1989), the accumulation of the reactive oxygen species (ROS) (Matsushima et al. 1998), and the activation of caspase (Kaushal et al. 2001). Previous studies have suggested several possible mechanisms for the vestibulotoxicity of cisplatin as follows:

\section{Cisplatin-induced oxidative stress}

Monroe et al (2018) reported that after $100 \mu \mathrm{M}$ cisplatin treatment, the ROS increased dramatically in saccular and utricular tissues of zebrafish. The release of ROS was sharply elevated as cisplatin's concentrations increased to $500 \mu \mathrm{M}$ (Monroe et al. 2018). The generation of ROS in the vestibular epithelium caused by cisplatin has been reported to cause vestibular HCs to undergo apoptosis (Tian et al. 2013).

Furthermore, the concentrations of lipid peroxidation (LPO) and nitric oxide (NO) increased significantly after cisplatin treatment (Cheng et al. 2006), which may impair the structure of the cell's membrane, inhibiting the activation of $\mathrm{Na}^{+}-\mathrm{K}^{+}$ATPase and $\mathrm{Ca}^{2+}$ ATPase of the ampullae of guinea pigs (Cheng et al. 2006). In the vestibular apparatus, $\mathrm{Na}^{+}-\mathrm{K}^{+}$ ATPase and $\mathrm{Ca}^{2+}$ ATPase, which are of importance for the transport of $\mathrm{K}^{+}$and $\mathrm{Ca}^{2+}$ respectively, can regulate endolymphatic ion distribution and endolymphatic volume. The decrease in $\mathrm{Na}^{+}-\mathrm{K}^{+}$ATPase's activity can lead to a reduction in $\mathrm{K}^{+}$concentration in the endolymph (Wangemann 2002). The reduction of $\mathrm{Ca}^{2+}$ ATPase's activity can alter $\mathrm{Ca}^{2+}$ concentration in endolymph as well. $\mathrm{Ca}^{2+}$ is a prerequisite for the electrical conductivity of $\mathrm{HCs}$ and requires exact regulation (Hudspeth 1985). As a result, the physiologic function of vestibular HCs might be affected, as two significant ions, $\mathrm{K}^{+}$and $\mathrm{Ca}^{2+}$, get disordered in the endolymph where $\mathrm{HCs}$ live and function.

It was reported that iNOS was found in the vestibular end organs after exposure to cisplatin (Watanabe et al. 2000). iNOS can catalyze an insufficient amount of NO, which can react with superoxide and produce peroxynitrites with strong oxidative effects and cytotoxic effects on tissues (Cazevieille et al. 1993; Huie and Padmaja 1993). Therefore, the author concluded that the expression of iNOS and the consecutive unphysiological high NO level might be involved in the vestibular toxicity of cisplatin (Watanabe et al. 2000).

\section{Caspase- and caspase-independent cell death pathway}

Two signal pathways have been studied to be involved in cisplatin-induced vestibular HC loss. In the vestibular organs exposed to cisplatin, caspase- 3 was detected within the cytoplasm of HCs in the utricle, saccule, and ampullae (Wu et al. 2017), and $24 \mathrm{~h}$ after cisplatin administration, the expression of caspase- 1 and caspase- 3 elevated remarkably in both HCs and supporting cells in the utricle of mice (Zhang et al. 2003). The caspase- 3 is of significance in the activation of caspase cascade-induced cell apoptosis (Watanabe et al. 
2001). Another study showed that it was the p53 that mediated the activation of caspase- 3 induced by cisplatin (Zhang et al. 2003).

The caspase-independent apoptotic pathway is also related to the loss of vestibular HCs. The apoptosis-inducing factor (AIF) is a kind of mitochondrial protein, which exists only between the inner membrane and the outer membrane of mitochondria under physiological conditions (Candé et al. 2002a). After exposure to cisplatin for seven days, AIFs could be found in the nucleus of vestibular HCs (Wu et al. 2017), and the rate of AIF nuclear translocation was noticeably increased in vestibular HCs (Wu et al. 2017). After being released from mitochondria, AIFs, capable of binding DNA in the nucleus, can activate the caspase-independent apoptotic pathway when they transfer to the nucleus (Candé et al. 2002a, b).

Some studies have found that some apoptosis-related gene expressions induced by cisplatin increased in HEI-OC1 cell lines (a cochlear hair cell line), including cytochrome c, c-Jun N-terminal kinase (JNK), Bcl-xL, and Bax (Tian et al. 2013). However, it remains unclear whether the activation of these pathways contributes to the loss of vestibular HCs.

\section{Proinflammatory cytokine expression}

Many studies have demonstrated that immune responses can affect vestibular function (Ma et al. 2000). The immune response in the inner ear is crucial for preventing microbial infections, but the increase of cytokines caused by immune responses can damage the inner ear tissue, leading to permanent vestibular dysfunction (Rahman et al. 2001) and hearing loss (Ryan et al. 2002).

Mitogen-activated protein kinases (MAPKs), including JNK and MEK/ERK, can be activated by cisplatin in the vestibular apparatus of mice (Kim et al. 2008). The phosphorylation of JNK and ERK can induce the nuclear translocation and transcriptional activation of NF-kB, one of the critical factors that can regulate the expression of proinflammatory cytokines, thus leading to the mRNA expression and secretion of proinflammatory cytokines. These cytokines include IL- $1 b$, IL-6, and TNF- $\alpha$ and may lead to cisplatin-mediated apoptosis of vestibular HCs (Kim et al. 2008).

\section{Aminoglycoside}

Aminoglycosides (AGs), such as neomycin, gentamicin, and streptomycin, being among the most commonly used antibiotics worldwide, are considered to be ototoxic (Selimoglu 2007). In human beings, gentamicin is thought to be more vestibulotoxic than cochleotoxic (Kim et al. 2013). Compare with cisplatin, aminoglycosides might affect the vestibular system much higher likelihood (Schacht et al. 2012).
The application of AGs can result in a dramatic loss of vestibular HCs (May et al. 2013; Nagato et al. 2018; Yoshida et al. 2015). With the dose of AGs elevated, the rate of vestibular HC loss increased (Lee et al. 2014; Park et al. 2007; Taleb et al. 2008; Yousaf et al. 2018). Apart from the decrease in vestibular $\mathrm{HC}$ density, there are also changes at the subcellular level, such as hydropic and vacuolar degeneration of vestibular HCs (Selimoğlu et al. 2003). Regarding $\mathrm{HC}$ death caused by ototoxic drugs, cisplatin mainly induces apoptosis, whereas AGs seem to induce both apoptosis and necrosis (Schacht et al. 2012). Some researchers have postulated that apoptosis might occur during the early three days, while vestibular $\mathrm{HC}$ death during the latter days may be attributed to necrosis (Hong et al. 2006). Distinct regions of vestibular organs and different $\mathrm{HC}$ subtypes have different sensitivity to AGs. The loss of vestibular HCs induced by gentamicin is mainly involved in type I hair cells (Kim et al. 2013; Qian et al. 2021), and HCs in the striolar region are more vulnerable to neomycin than HCs in the extra-striolar region (Cunningham et al. 2002).

The mechanisms of cochlear toxicity of AGs have been illuminated, but vestibular toxicity mechanisms of AGs have been relatively little studied. Existing studies have shown that the ROS is the critical mediator of aminoglycosideinduced vestibular toxicity. AGs exposure can give rise to the formation of ROS in HCs (Hirose et al. 1997), which activates the JNK (Sugahara et al. 2006; Yu et al. 2004). The activation of JNK can lead to the release of cytochrome c from mitochondria into the cytoplasm, and cytochrome $\mathrm{c}$ lies upstream of the activation of caspase-9, which can trigger the activation of downstream caspase-3 (Cunningham et al. 2002). Lee et al. pointed out that HC death caused by aminoglycosides may involve a mechanism that the ROS suddenly increase intracellular $\mathrm{Ca}^{2+}$ concentration and open up the mitochondrial permeability transition (MPT) pore. Increased intracellular $\mathrm{Ca}^{2+}$ levels and the MPT opening are essential elements in the apoptosis caused by aminoglycoside. The resulting cell apoptosis can, in turn, increase intracellular $\mathrm{Ca}^{2+}$, thus creating a vicious cycle (Lee et al. 2014).

Other studies have suggested that the increased intracellular $\mathrm{Ca}^{2+}$ levels in vestibular HCs induced by AGs might be the consequence of blocking voltage-gated $\mathrm{Ca}^{2+}$ channels (Ding et al. 2002). Then, the increased intracellular $\mathrm{Ca}^{2+}$ can activate calpains, promoting the degradation of kinases, proteins, and transcription factors (Bartus et al. 1995). Overactivation of calpain will eventually result in vestibular HC death (Kim et al. 2013).

Zhang et al. showed that AIFs were elevated after gentamicin application in vestibular tissue of rats. The amount of AIFs in mitochondria decreased, while the number of AIFs in the cytoplasm increased, suggesting that AIF might be one of the essential factors in apoptosis of vestibular HCs induced by gentamicin (Zhang et al. 2012). 


\section{Aging}

Many histopathological studies of human vestibular sensory epithelia have indicated that vestibular HCs degenerate with age (Engström et al. 1977; Lopez et al. 2005; Richter 1980). The HC densities of all five vestibular end organs decreased in subjects with an average age of 84 and 94 years old (Rauch et al. 2001). The declination rate of Type I HCs in the crista ampullaris was higher than that in the macula, whereas there was the same declination rate of type II HCs in all five vestibular end organs(Rauch et al. 2001). However, through an unbiased stereological analysis of 10 subjects with an average age of 82, no age-related $\mathrm{HC}$ loss was found in the utricle (Gopen et al. 2003). Differences in the degree of $\mathrm{HC}$ degeneration can also be found in different peripheral vestibular apparatus. The loss of HCs in the crista ampullaris was greater than that in the macula (Anniko 1983), and there was a more significant loss of $\mathrm{HC}$ in the saccule than that in the utricle (Rosenhall 1973). There are also some underlying age-related changes in vestibular HCs, including the loss and disorder of cilia, the accumulation of lipofuscin pigments, the appearance of intracellular vesicles, the disruption of the cuticular plate (Anniko 1983; Richter 1980), as well as some mitochondrial changes, such as the abnormal morphology and the increased number of mitochondria, and the disintegration of mitochondrial crista (Baloh et al. 1997).

Age-related hearing loss is now recognized as a disorder caused by a combination of many internal genetic factors and external environmental factors (Ohlemiller 2009; Yamasoba et al. 2013). By comparison, little known is about mechanisms behind the changes of HCs in vestibular sensory epithelia. However, based on multiple relevant research, some possible reasons have been suggested. With aging, the number of mtDNA copies has been reported to decrease by $50-70 \%$, and gene expression associated with oxidative phosphorylation (OXPHOS) is also reduced. Based on these facts above, one reason may be that vestibular HCs that are considered to be heavily dependent on OXPHOS for ATP (Spinelli et al. 2012) may become more sensitive to ototoxic factors due to this potential energy crisis (Bigland et al. 2018). Other researchers have suggested that the accumulation of calcium and ROS might be of significance in the age-related injury of vestibular HCs (Brosel et al. 2016). This hypothesis was supported by the following facts: (1) Aging mice showed a decrease in several proteins, including TRPV5, TRPV6, and Klotho (Tanaka et al. 2012). These proteins are significant to $\mathrm{Ca}^{2+}$ metabolism in vestibular and cochlear tissues, and the normal function of HCs depends on low concentrations of calcium in the endolymph. Besides, Klotho can attenuate oxidative stress (Yamoah et al. 1998); (2) With age, some oxidative stress markers such as nitrotyrosine and hydroxynonenal in the inner ear increased. Meanwhile, SOD2, one of the important antioxidant enzymes, decreased (Yamoah et al. 1998). This oxidative stress may result in mitochondrial dysfunction and result from the continuously increased deletions of mitochondrial DNA, leading to a vicious cycle (Crawley and Keithley 2011; Markaryan et al. 2008).

\section{Genetic factors}

Genetic factors can contribute to vestibular HC loss. The MyoXVa: whirlin: Eps8 complex at the tips of stereocilia is critical for the elongation of stereocilia (Manor et al. 2011). The role of whirlin is to help the MyoXVa: Eps8 complex assemble properly and increase its stability (Manor et al. 2011). Eps 8 directly regulates the role of whirlin in stereocilia (Manor et al. 2011). Myosin VI functions as a connection between stereocilia and the cuticular plate (Hasson et al. 1997). As a result, the mutation of genes related to stereociliary function can result in the damage of vestibular HCs. The vestibular HCs of Eps8-KO mice showed significantly shorter stereocilia (Tavazzani et al. 2016). The whirler mouse, a model of human Usher syndrome, which cannot produce functional whirlin proteins encoded by WHRN in the inner ear (Mathur et al. 2015b), manifested abnormally shortened stereocilia of both cochlear and vestibular HCs (Holme et al. 2002; Mburu et al. 2003). Furthermore, both Dfnb31neo/neo mice, which has a deletion between Dfnb31(WHRN) exons 6 and 9, and Dfnb31wi/wi mice, which has a mutation in Dfnb31 exon 1, were characterized by shortened stereocilia of the macula (Mathur et al. 2015a). Tur/Tur mutant mice, which has a point mutation in exon 8 of the Myo6 gene, showed a significant reduction of HCs in the three cristae. Their stereociliary bundles seemed to be elongated, distorted, and disordered in the macula (Wong et al. 2016). It has been observed that mice with a deletion of exon 5 in the Myo6 gene exhibited elongated and fused stereocilia at P2, and even complete absence of stereocilia at eight weeks (Williams et al. 2013).

Apart from this, some studies also found additional genes, without which vestibular HCs cannot maintain in a normal state. Ablation of the function of prosaposin (a precursor of four glycoprotein activators for lysosomal hydrolases) led to the overgrowth of afferent and efferent neurons below vestibular HCs and these excess neurons can destroy HCs and supporting cells in the vestibular end organs of mice (Akil and Lustig 2012). After deletion of Brg1, an ATPase subunit of a chromatin-remodeling complex, vestibular HCs of mice exhibited fused and elongated stereocilia bundles, and most $\mathrm{HCs}$ with fused stereociliary bundles did not have a cuticular plate (Akil and Lustig 2012). A mutation in the SLC26A4 gene can cause extensive $\mathrm{HC}$ loss and degeneration in the saccule, utricle, and crista of mice (Lu et al. 2011). Mice with a defect of 
Brn-3c showed a substantial loss of $\mathrm{HCs}$ in cochlear and vestibular epithelia during the third trimester and early postnatal period (Xiang et al. 1997).

\section{Protection strategies of vestibular hair cells}

Currently, practical strategies for preventing the loss or damage of vestibular HCs mainly focus on pharmacotherapy and gene therapy.

\section{Pharmacotherapies}

\section{Heat shock protein}

Various kinds of cellular and environmental stress can induce the expression of heat shock proteins (HSPs). The induction of HSPs is a ubiquitous and highly conserved stress response that can inhibit apoptosis in numerous systems (Martindale and Holbrook 2002). Some studies have argued that heat shock-induced HSPs inhibit aminoglycoside-induced (Taleb et al. 2008) and cisplatininduced (Baker et al. 2015) HC death in mouse utricle. Both HSP 70 and its major heat shock transcription factor, HSF1, are necessary for performing this protective effect (Baker et al. 2015), and some studies have observed that HSP70 is mainly secreted by supporting cells, not by HCs in heat shock responses in the inner ear (May et al. 2013). Although constitutive expression of HSP70 brings about a robust protective effect against HC loss from aminoglycoside treatment (Taleb et al. 2008), it provides only modest protection against $\mathrm{HC}$ death induced by cisplatin (Baker et al. 2015). Overexpression of HSP70 inhibits vestibular HC death from ototoxicity as well (May et al. 2013). Furthermore, some factors, which can induce HSP70 in the vestibular end organs, such as geranylgeranylacetone (GGA) (Nagato et al. 2018; Takumida and Anniko 2005) and pifithin- $\mu$ (Ryals et al. 2018), also protect vestibular HCs from aminoglycoside-induced death. HSP32 is another heat shock protein that may prevent vestibular toxicity induced by cisplatin, and its protective effect is mediated by resident macrophages (Baker et al. 2015). Celastrol, a traditional Chinese medicine, can induce HSP32 through activating the Nrf2-transcription factor (Francis et al. 2011), and HSP32 can inhibit pro-apoptotic JNK activation and HCs death (Francis et al. 2011). In summary, these studies above underscored the beneficial effects of HSP70, HSP32, and their inducer against the ototoxic drug-induced vestibular HC loss. However, mechanisms by which HSP protects against vestibular HC loss need further investigation in the future.

\section{Antioxidant treatment}

For protecting vestibular HCs from damage, antioxidants have potential therapeutic effects. Metformin, which can greatly reduce the release of ROS induced by gentamicin, inhibited gentamicin-induced apoptosis of HCs in rat utricle (Lee et al. 2014). Renexin (RXN), a combination of Ginkgo biloba extract (GbE) and Cilostazol (CS), noticeably attenuated cisplatin-mediated ROS production in HEI-OC1 cells (Tian et al. 2013). When combined with cisplatin, RXN kept stereocilia in the rat utricle and saccule preserved and well-shaped (Lee et al. 2014). Owing to a deletion mutation in the $\gamma$-glutamyl transferase- 1 gene, dwarf gray mice cannot encode an enzyme essential for glutathione resynthesis, giving rise to the loss of vestibular $\mathrm{HCs}$ and large vacuoles in vestibular HCs of dwarf gray mice (Ding et al. 2016). Hydrogen gas can effectively protect vestibular HCs against morphological and functional damage caused by antimycin A by reducing ROS (Taura et al. 2010). Collectively, these studies indicated that antioxidants are a promising approach for rescuing vestibular $\mathrm{HC}$ loss caused by ototoxic drugs.

Other factors have been shown to reduce cisplatininduced ROS in vestibular organs, including D-methionine (Cheng et al. 2006), which can reduce cisplatin-induced LPO and NO in the vestibular HCs of guinea pigs, and EF24(bis[(2-fluorophenyl)methylene]-4-piperidinone) (Monroe et al. 2018) which can decrease ROS release by cisplatin treatment in the maculae of zebrafish. However, further investigation is required to understand the protective effects of D-methionine and EF-24 on vestibular HCs. Both the L-NG-nitroarginine methyl ester (L-NAME) (Takumida and Anniko 2002; Takumida et al. 2003), a non-special NOS inhibitor, and D-methionine (Takumida et al. 2003), a radical scavenger, both increased the survival rate of vestibular HCs after the treatment of gentamicin. Some studies (Hoshino et al. 2011; Kim et al. 2015) have noted that $\mathrm{Nrf2}$, an antioxidant factor, can prevent cochlear HC loss from ototoxic drugs and age-related damage. Kumiko et al. demonstrated that Nrf2-IR is also present in HCs and supporting cells of five vestibular end organs (Hosokawa et al. 2018). However, the protective effect of Nrf2 in vestibular HCs needs further explorations.

\section{Inhibition of programmed cell death}

The use of diverse antiapoptotic agents to manipulate intrinsic cell death is also a promising intervention for protecting vestibular HCs. Several scientific research has reported that the MAPK/JNK pathway plays an essential role in the death of cochlear HCs and vestibular HCs caused by aminoglycosides (Sugahara et al. 2006). In animal models, blocking this pathway with the JNK inhibitor CEP11004 considerably protected utricular HCs treated with intermediate doses of 
neomycin (Sugahara et al. 2006), but it did not show any protective effect against high doses of neomycin-induced vestibular HC death (Sugahara et al. 2006). A study disclosed that allicin inhibited caspase-dependent and caspase-independent apoptosis pathways and protected cisplatin-induced vestibular HC loss (Wu et al. 2017). Inhibition of caspase activation by the administration of the pan-caspase inhibitor zVAD promoted the survival of vestibular HCs after aminoglycoside treatment in chicks (Matsui et al. 2003).

\section{Growth factors}

Insulin-like growth factor-1 (IGF-1) is considered to be one of the essential regulators for the growth and development of the inner ear (Varela-Nieto et al. 2004), and many studies have shown its vestibular protective effect for preventing vestibular HC loss by aminoglycoside exposure (Angunsri et al. 2011; Park et al. 2007; Yoshida et al. 2015). Extensive loss of HCs in the utricle caused by neomycin was rescued by IGF-1 treatment (Angunsri et al. 2011). Parallel to IGF-I, IGFBP(insulin-like growth factor binding protein)-4 and IGFBP-5 are significantly protective (Park et al. 2007). IGF-I, IGFBP-4, IGFBP -5, and the combination of IGF-I and IGFBP-5 increased HC survival in mouse utricle after neomycin application (Park et al. 2007). However, the combination of IGFBP-4 and IGF-I is less effective than applying each one alone (Park et al. 2007). The SSSR, which is the minimum peptide of IGF-1, and the combination of SSSR and FGLM-NH2 which is the smallest peptide of SP, have also been reported to be protective against neomycininduced vestibulotoxicity (Yoshida et al. 2015). However, the mechanisms underlying those protective effects remain unclear, but there are some hypotheses: (1) Unlike HCs of cochlear epithelia, vestibular HCs of mammals can regenerate to some extend (Taura et al. 2006), and IGF-1 may facilitate this regeneration (Kopke et al. 2001) to protect vestibular HCs (Park et al. 2007); (2) Aminoglycoside-induced HC loss is associated with activation of JNK (Zheng et al. 1997) and IGF-1 in the inner ear can modulate JNK activation, thereby inhibiting aminoglycosides-induced HC death. However, additional experiments are required to confirm these hypotheses. Treatment with brain-derived neurotrophic factor (BDNF) also has protective effects against vestibular HC degeneration by gentamicin (Takumida and Anniko 2002; Takumida et al. 2003).

\section{Other protective agents and molecules}

Excepted from what has been stated above, there are other treatments for vestibular HC loss. Leupeptin, a calpain inhibitor, noticeably inhibited gentamicin-induced density reduction of HCs in the utricle and crista ampullaris of mice induced by gentamicin (Ding et al. 2002). Tacrine has also been elucidated to protect the utricular HCs of mice against neomycin (Ou et al. 2009). When pretreated with tacrine, $\mathrm{HCs}$ from both striolar and extrastriola regions were retained after applying neomycin. P2X2 purinergic receptors in vestibular transitional cells (Lee et al. 2001) have been reported to carry cation currents, thereby protecting utricular HCs during increased stimulation intensity (Jeong et al. 2020). Korean red ginseng has been proven to protect against age-related $\mathrm{HC}$ loss in five periphery vestibular organs (Tian et al. 2014).

\section{The combination of different pharmacotherapies}

It has been indicated in some studies that the protective effect of a drug combination is better than using one drug alone. Treatment with combinations of L-NAME + BDNF (Takumida and Anniko 2002; Takumida et al. 2003), L-NAME + leupeptin (Takumida et al. 2003), and D-methionine + BDNF (Takumida et al. 2003) can save more vestibular $\mathrm{HC}$ after exposure to gentamicin than using any one of them alone. So researchers suggested that the combinations of ROS scavengers with neurotrophic factors or ROS scavengers with calpain inhibitors may yield greater therapeutic effects for vestibular diseases (Takumida et al. 2003).

\section{Gene therapy}

Gene therapy refers to the insertion of foreign genes into the appropriate receptor cells of patients through gene transfer technology, so that the product produced by extraneous genes can achieve a desired therapeutic outcome. It provides an opportunity to repair mutated genes before birth, thus antagonizing the morphological or functional insufficiency of vestibular HCs caused by some congenital gene-related diseases. At present, in vestibular HC protection, gene therapy has been relatively widely used in the treatment of Usher syndrome. Delivering gene products to vestibular HCs via adeno-associated virus (AAV), the distorted stereociliary bundles due to the Whirlin mutation in an Usher2d mouse model was successfully restored, and the survival rate of HCs increased in both the cochlea and utricle (Chien et al. 2016; Isgrig et al. 2017). Lentz et al. saved vestibular and cochlear HCs of Usher1c mouse by correcting pre-mRNA splicing defects in Usher1c mouse model with antisense oligonucleotides (Lentz et al. 2013). Gene therapy mediated by AV2/Anc80L65 restored mechanical transduction of vestibular HCs in another Ush1c mutant mouse model (Pan et al. 2017). The stereociliary length of whirler mice was successfully restored after injecting AAV8-whirlin into posterior semicircular canals (Isgrig et al. 2017). 


\section{Conclusions}

For the past few years, a majority of studies on vestibular HCs have mainly focused on the development of multiple therapeutic strategies to protect vestibular HCs from destruction and reverse vestibular dysfunction. Numerous vestibular protective measurements, consisting of heat shock proteins and their stimulants, antioxidants, antiinflammatory agents, manipulation of intrinsic pathways of cell death, growth factors, and gene therapy, are likely to provide an impetus for the comprehensive development of vestibular HC protection in the future. Furthermore, understanding the mechanisms of vestibular HC toxicity caused by distinct factors can also help develop effective treatments aiming at these mechanisms.

In addition to the protective agents targeting the mechanisms of vestibular $\mathrm{HC}$ damage mentioned in this review, such as antiapoptotic and antioxidant drugs, to our knowledge, some mechanisms that cause vestibular HC damage have not been reported with targeted drugs. In the future, attempts can be made in these mechanisms, such as whether antagonizing the increase of inflammatory cytokines induced by cisplatin can save cisplatininduced vestibular $\mathrm{HC}$ loss; Whether overexpression of mtDNA can prevent energy deficiency caused by the loss of mtDNA due to vestibular $\mathrm{HC}$ aging.

Additionally, compared with vestibular HCs, the factors leading to the loss of cochlear HCs and their mechanisms, as well as the corresponding therapeutic strategies, have been relatively clarified. With histological correlations between cochlear and vestibular HCs, we can verify whether these factors, mechanisms, and treatments have proven to be effective in cochlear HCs by multiple existing studies but have not yet been applied to vestibular HCs, can be used to vestibular HCs in the future. For example, whether noise and viral infection that damage cochlear HCs will lead to vestibular HC loss; Whether the PI3KAkt pathway causing the apoptosis of cochlear HCs is involved in the apoptosis of vestibular HCs, and whether the mutation in Pou $4 \mathrm{f} 3$ gene and mtDNA that increases the susceptibility to ototoxic drugs of cochlear HCs can increase the sensitivity to ototoxic drugs of vestibular $\mathrm{HC}$; Whether some antioxidants such as $\mathrm{N}$-acetylcysteine, MitoQ, Nrf2, and antiapoptotic agents, such as KNUA002, DUSP1, which have been elucidated to protect cochlear $\mathrm{HCs}$, can perform similar protective effects on vestibular HCs. Moreover, whether some strategies used to preserve cochlear HCs, such as chemical modification of ototoxic drugs, application of non-coding RNA, inhibition of the uptake of toxic factors, regulation of autophagy of HCs, and manipulation of epigenetics such as methylation and acetylation, can effectively protect vestibular HCs.
Furthermore, with the progress of emerging technologies, novel methods can be used for further investigation. For example, scRNA-seq can be used to identify more genes regulating vestibular HC damage; Epigenomics and proteomics can be used to ascertain the mechanisms of various injury factors leading to vestibular HC loss; The library of integrated cellular signatures and bioactive compounds can be used to recognize new otoprotective agents; CRISPR/Cas9 can be used to treat gene-related vestibular HC degeneration.

Author contributions LJ wrote the original manuscript. $\mathrm{ZZ}$ and $\mathrm{YH}$ contributed to the conception of this research. All authors contributed to drafting the manuscript and approve the final version. They also agree to the accuracy and integrity of the manuscript.

Funding This work was supported by Grants from the the National Natural Science Foundation of China (Nos. 82071045, 81870728), and Sponsored by Shanghai Rising-Star Program (19QA1401800).

\section{Declarations}

Conflict of interest The authors declare no competing financial interests.

Open Access This article is licensed under a Creative Commons Attribution 4.0 International License, which permits use, sharing, adaptation, distribution and reproduction in any medium or format, as long as you give appropriate credit to the original author(s) and the source, provide a link to the Creative Commons licence, and indicate if changes were made. The images or other third party material in this article are included in the article's Creative Commons licence, unless indicated otherwise in a credit line to the material. If material is not included in the article's Creative Commons licence and your intended use is not permitted by statutory regulation or exceeds the permitted use, you will need to obtain permission directly from the copyright holder. To view a copy of this licence, visit http://creativecommons.org/licenses/by/4.0/.

\section{References}

Agrawal Y, Carey JP, Della Santina CC, Schubert MC, Minor LB (2009) Disorders of balance and vestibular function in US adults: data from the National Health and Nutrition Examination Survey, 2001-2004. Arch Intern Med 169(10):938-944

Akil O, Lustig LR (2012) Severe vestibular dysfunction and altered vestibular innervation in mice lacking prosaposin. Neurosci Res 72(4):296-305

Angunsri N, Taura A, Nakagawa T et al (2011) Insulin-like growth factor 1 protects vestibular hair cells from aminoglycosides. NeuroReport 22(1):38-43

Anniko M (1983) The aging vestibular hair cell. Am J Otolaryngol 4(3):151-160

Baker TG, Roy S, Brandon CS et al (2015) Heat shock protein-mediated protection against Cisplatin-induced hair cell death. J Assoc Res Otolaryngol 16(1):67-80 
Baloh RW, Lopez I, Beykirch K, Ishiyama A, Honrubia V (1997) Clinical-pathologic correlation in a patient with selective loss of hair cells in the vestibular endorgans. Neurology 49(5):1377-1382

Bartus RT, Elliott PJ, Hayward NJ et al (1995) Calpain as a novel target for treating acute neurodegenerative disorders. Neurol Res 17(4):249-258

Bigland MJ, Brichta AM, Smith DW (2018) Effects of ageing on the mitochondrial genome in rat vestibular organs. Curr Aging Sci 11(2):108-117

Brosel S, Strupp M (2019) The vestibular system and ageing. Subcell Biochem 91:195-225

Brosel S, Laub C, Averdam A, Bender A, Elstner M (2016) Molecular aging of the mammalian vestibular system. Ageing Res Rev 26:72-80

Burns JC, Stone JS (2017) Development and regeneration of vestibular hair cells in mammals. Semin Cell Dev Biol 65:96-105

Candé C, Cecconi F, Dessen P, Kroemer G (2002a) Apoptosis-inducing factor (AIF): key to the conserved caspase-independent pathways of cell death? J Cell Sci 115(Pt 24):4727-4734

Candé C, Cohen I, Daugas E et al (2002b) Apoptosis-inducing factor (AIF): a novel caspase-independent death effector released from mitochondria. Biochimie 84(2-3):215-222

Cazevieille C, Muller A, Meynier F, Bonne C (1993) Superoxide and nitric oxide cooperation in hypoxia/reoxygenation-induced neuron injury. Free Radic Biol Med 14(4):389-395

Cheng PW, Liu SH, Young YH, Lin-Shiau SY (2006) D-Methionine attenuated cisplatin-induced vestibulotoxicity through altering ATPase activities and oxidative stress in guinea pigs. Toxicol Appl Pharmacol 215(2):228-236

Chien WW, Isgrig K, Roy S et al (2016) Gene therapy restores hair cell stereocilia morphology in inner ears of deaf whirler mice. Mol Ther 24(1):17-25

Crawley BK, Keithley EM (2011) Effects of mitochondrial mutations on hearing and cochlear pathology with age. Hear Res 280(1-2):201-208

Cunningham LL, Brandon CS (2006) Heat shock inhibits both aminoglycoside- and cisplatin-induced sensory hair cell death. J Assoc Res Otolaryngol 7(3):299-307

Cunningham LL, Cheng AG, Rubel EW (2002) Caspase activation in hair cells of the mouse utricle exposed to neomycin. J Neurosci 22(19):8532-8540

Ding D, Stracher A, Salvi RJ (2002) Leupeptin protects cochlear and vestibular hair cells from gentamicin ototoxicity. Hear Res 164(1-2):115-126

Ding D, Jiang H, Chen GD et al (2016) N-acetyl-cysteine prevents age-related hearing loss and the progressive loss of inner hair cells in $\gamma$-glutamyl transferase 1 deficient mice. Aging (albany NY) 8(4):730-750

Ding D, Jiang H, Zhang J et al (2018) Cisplatin-induced vestibular hair cell lesion-less damage at high doses. J Otol 13(4):115-121

Engström H, Ades HW, Engström B, Gilchrist D, Bourne G (1977) Structural changes in the vestibular epithelia in elderly monkeys and humans. Adv Otorhinolaryngol 22:93-110

Francis SP, Kramarenko II, Brandon CS et al (2011) Celastrol inhibits aminoglycoside-induced ototoxicity via heat shock protein 32 . Cell Death Dis. 2:e195

Gopen Q, Lopez I, Ishiyama G, Baloh RW, Ishiyama A (2003) Unbiased stereologic type I and type II hair cell counts in human utricular macula. Laryngoscope 113(7):1132-1138

Hasson T, Gillespie PG, Garcia JA et al (1997) Unconventional myosins in inner-ear sensory epithelia. J Cell Biol 137(6):1287-1307

Hirose K, Hockenbery DM, Rubel EW (1997) Reactive oxygen species in chick hair cells after gentamicin exposure in vitro. Hear Res 104(1-2):1-14
Holme RH, Kiernan BW, Brown SD, Steel KP (2002) Elongation of hair cell stereocilia is defective in the mouse mutant whirler. J Comp Neurol 450(1):94-102

Hong SH, Park SK, Cho YS et al (2006) Gentamicin induced nitric oxide-related oxidative damages on vestibular afferents in the guinea pig. Hear Res 211(1-2):46-53

Hoshino T, Tabuchi K, Nishimura B et al (2011) Protective role of Nrf2 in age-related hearing loss and gentamicin ototoxicity. Biochem Biophys Res Commun 415(1):94-98

Hosokawa K, Hosokawa S, Ishiyama G, Ishiyama A, Lopez IA (2018) Immunohistochemical localization of $\mathrm{Nrf} 2$ in the human cochlea. Brain Res 1700:1-8

Hudspeth AJ (1985) The cellular basis of hearing: the biophysics of hair cells. Science 230(4727):745-752

Hudspeth AJ, Corey DP (1977) Sensitivity, polarity, and conductance change in the response of vertebrate hair cells to controlled mechanical stimuli. Proc Natl Acad Sci U S A 74(6):2407-2411

Huie RE, Padmaja S (1993) The reaction of no with superoxide. Free Radic Res Commun 18(4):195-199

Isgrig K, Shteamer JW, Belyantseva IA et al (2017) Gene therapy restores balance and auditory functions in a mouse model of usher syndrome. Mol Ther 25(3):780-791

Jeong J, Kim JY, Hong H et al (2020) P2RX2 and P2RX4 receptors mediate cation absorption in transitional cells and supporting cells of the utricular macula. Hear Res. 386:107860

Ji L, Zhai S (2018) Aging and the peripheral vestibular system. J Otol 13(4):138-140

Kaushal GP, Kaushal V, Hong X, Shah SV (2001) Role and regulation of activation of caspases in cisplatin-induced injury to renal tubular epithelial cells. Kidney Int 60(5):1726-1736

Khan S, Chang R (2013) Anatomy of the vestibular system: a review. NeuroRehabilitation 32(3):437-443

Kim HJ, So HS, Lee JH et al (2008) Role of proinflammatory cytokines in cisplatin-induced vestibular hair cell damage. Head Neck 30(11):1445-1456

Kim HJ, Lee JO, Koo JW, Kim JS, Ban J (2013) Gentamicin-induced bilateral vestibulopathy in rabbits: vestibular dysfunction and histopathology. Laryngoscope 123(11):E51-58

Kim SJ, Ho Hur J, Park C et al (2015) Bucillamine prevents cisplatininduced ototoxicity through induction of glutathione and antioxidant genes. Exp Mol Med. 47(2):e142

Kingma H, van de Berg R (2016) Anatomy, physiology, and physics of the peripheral vestibular system. Handb Clin Neurol 137:1-16

Kopke RD, Jackson RL, Li G et al (2001) Growth factor treatment enhances vestibular hair cell renewal and results in improved vestibular function. Proc Natl Acad Sci U S a 98(10):5886-5891

Lee JH, Chiba T, Marcus DC (2001) P2X2 receptor mediates stimulation of parasensory cation absorption by cochlear outer sulcus cells and vestibular transitional cells. J Neurosci 21(23):9168-9174

Lee JY, Lee SH, Chang JW et al (2014) Protective effect of metformin on gentamicin-induced vestibulotoxicity in rat primary cell culture. Clin Exp Otorhinolaryngol 7(4):286-294

Leibbrandt ME, Wolfgang GH, Metz AL, Ozobia AA, Haskins JR (1995) Critical subcellular targets of cisplatin and related platinum analogs in rat renal proximal tubule cells. Kidney Int 48(3):761-770

Lentz JJ, Jodelka FM, Hinrich AJ et al (2013) Rescue of hearing and vestibular function by antisense oligonucleotides in a mouse model of human deafness. Nat Med 19(3):345-350

Lopez I, Ishiyama G, Tang Y et al (2005) Regional estimates of hair cells and supporting cells in the human crista ampullaris. J Neurosci Res 82(3):421-431

Lu YC, Wu CC, Shen WS et al (2011) Establishment of a knock-in mouse model with the SLC26A4 c.919-2A>G mutation and characterization of its pathology. PLoS ONE 6(7):e22150 
Ma C, Billings P, Harris JP, Keithley EM (2000) Characterization of an experimentally induced inner ear immune response. Laryngoscope 110(3 Pt 1):451-456

Manor U, Disanza A, Grati M et al (2011) Regulation of stereocilia length by myosin XVa and whirlin depends on the actin-regulatory protein Eps8. Curr Biol 21(2):167-172

Markaryan A, Nelson EG, Hinojosa R (2008) Detection of mitochondrial DNA deletions in the cochlea and its structural elements from archival human temporal bone tissue. Mutat Res 640(1-2):38-45

Martindale JL, Holbrook NJ (2002) Cellular response to oxidative stress: signaling for suicide and survival. J Cell Physiol 192(1):1-15

Mathur PD, Vijayakumar S, Vashist D et al (2015a) A study of whirlin isoforms in the mouse vestibular system suggests potential vestibular dysfunction in DFNB31-deficient patients. Hum Mol Genet 24(24):7017-7030

Mathur PD, Zou J, Zheng T et al (2015b) Distinct expression and function of whirlin isoforms in the inner ear and retina: an insight into pathogenesis of USH2D and DFNB31. Hum Mol Genet 24(21):6213-6228

Matsui JI, Haque A, Huss D et al (2003) Caspase inhibitors promote vestibular hair cell survival and function after aminoglycoside treatment in vivo. J Neurosci 23(14):6111-6122

Matsushima H, Yonemura K, Ohishi K, Hishida A (1998) The role of oxygen free radicals in cisplatin-induced acute renal failure in rats. J Lab Clin Med 131(6):518-526

May LA, Kramarenko II, Brandon CS et al (2013) Inner ear supporting cells protect hair cells by secreting HSP70. J Clin Invest 123(8):3577-3587

Mburu P, Mustapha M, Varela A et al (2003) Defects in whirlin, a PDZ domain molecule involved in stereocilia elongation, cause deafness in the whirler mouse and families with DFNB31. Nat Genet 34(4):421-428

Merchant SN, Velázquez-Villaseñor L, Tsuji K et al (2000) Temporal bone studies of the human peripheral vestibular system. Normative vestibular hair cell data. Ann Otol Rhinol Laryngol Suppl. 181:3-13

Monroe JD, Millay MH, Patty BG, Smith ME (2018) The curcuminoid, EF-24, reduces cisplatin-mediated reactive oxygen species in zebrafish inner ear auditory and vestibular tissues. J Clin Neurosci 57:152-156

Nagato S, Sugahara K, Hirose Y et al (2018) Oral administration of geranylgeranylacetone to protect vestibular hair cells. Auris Nasus Larynx 45(3):412-416

Negishi-Oshino R, Ohgami N, He T et al (2019) Heat shock protein 70 is a key molecule to rescue imbalance caused by low-frequency noise. Arch Toxicol 93(11):3219-3228

Ohlemiller KK (2009) Mechanisms and genes in human strial presbycusis from animal models. Brain Res 1277:70-83

Ou HC, Cunningham LL, Francis SP et al (2009) Identification of FDA-approved drugs and bioactives that protect hair cells in the zebrafish (Danio rerio) lateral line and mouse (Mus musculus) utricle. J Assoc Res Otolaryngol 10(2):191-203

Pan B, Askew C, Galvin A et al (2017) Gene therapy restores auditory and vestibular function in a mouse model of Usher syndrome type 1c. Nat Biotechnol 35(3):264-272

Park JY, Park YH, Shin DH, Oh SH (2007) Insulin-like growth factor binding protein (IGFBP)-mediated hair cell survival on the mouse utricle exposed to neomycin: the roles of IGFBP-4 and IGFBP-5. Acta Otolaryngol Suppl 558:22-29

Qian X, Ma R, Wang X et al (2021) Simultaneous gentamicin-mediated damage and Atoh1 overexpression promotes hair cell regeneration in the neonatal mouse utricle. Exp Cell Res. 398(1):112395
Rahman MU, Poe DS, Choi HK (2001) Autoimmune vestibulo-cochlear disorders. Curr Opin Rheumatol 13(3):184-189

Rauch SD, Velazquez-Villaseñor L, Dimitri PS, Merchant SN (2001) Decreasing hair cell counts in aging humans. Ann N Y Acad Sci 942:220-227

Richter E (1980) Quantitative study of human Scarpa's ganglion and vestibular sensory epithelia. Acta Otolaryngol 90(3-4):199-208

Ries F, Klastersky J (1986) Nephrotoxicity induced by cancer chemotherapy with special emphasis on cisplatin toxicity. Am J Kidney Dis 8(5):368-379

Rosenhall U (1973) Degenerative patterns in the aging human vestibular neuro-epithelia. Acta Otolaryngol 76(2):208-220

Ryals M, Morell RJ, Martin D et al (2018) The inner ear heat shock transcriptional signature identifies compounds that protect against aminoglycoside ototoxicity. Front Cell Neurosci 12:445

Ryan AF, Harris JP, Keithley EM (2002) Immune-mediated hearing loss: basic mechanisms and options for therapy. Acta Otolaryngol Suppl 548:38-43

Schacht J, Talaska AE, Rybak LP (2012) Cisplatin and aminoglycoside antibiotics: hearing loss and its prevention. Anat Rec (hoboken) 295(11):1837-1850

Selimoglu E (2007) Aminoglycoside-induced ototoxicity. Curr Pharm Des 13(1):119-126

Selimoğlu E, Kalkandelen S, Erdoğan F (2003) Comparative vestibulotoxicity of different aminoglycosides in the Guinea pigs. Yonsei Med j 44(3):517-522

Spinelli KJ, Klimek JE, Wilmarth PA et al (2012) Distinct energy metabolism of auditory and vestibular sensory epithelia revealed by quantitative mass spectrometry using MS2 intensity. Proc Natl Acad Sci U S a 109(5):E268-277

Sugahara K, Rubel EW, Cunningham LL (2006) JNK signaling in neomycin-induced vestibular hair cell death. Hear Res 221(1-2):128-135

Sugiyama S, Hayakawa M, Kato T et al (1989) Adverse effects of antitumor drug, cisplatin, on rat kidney mitochondria: disturbances in glutathione peroxidase activity. Biochem Biophys Res Commun 159(3):1121-1127

Takumida M, Anniko M (2002) Brain-derived neurotrophic factor and nitric oxide synthase inhibitor protect the vestibular organ against gentamicin ototoxicity. Acta Otolaryngol 122(1):10-15

Takumida M, Anniko M (2005) Heat shock protein 70 delays gentamicin-induced vestibular hair cell death. Acta Otolaryngol 125(1):23-28

Takumida M, Anniko M, Shimizu A, Watanabe H (2003) Neuroprotection of vestibular sensory cells from gentamicin ototoxicity obtained using nitric oxide synthase inhibitors, reactive oxygen species scavengers, brain-derived neurotrophic factors and calpain inhibitors. Acta Otolaryngol 123(1):8-13

Taleb M, Brandon CS, Lee FS et al (2008) Hsp70 inhibits aminoglycoside-induced hair cell death and is necessary for the protective effect of heat shock. J Assoc Res Otolaryngol 9(3):277-289

Tanaka C, Coling DE, Manohar S et al (2012) Expression pattern of oxidative stress and antioxidant defense-related genes in the aging Fischer 344/NHsd rat cochlea. Neurobiol Aging 33(8):1842.e1841-1814

Taura A, Kojima K, Ito J, Ohmori H (2006) Recovery of hair cell function after damage induced by gentamicin in organ culture of rat vestibular maculae. Brain Res 1098(1):33-48

Taura A, Kikkawa YS, Nakagawa T, Ito J (2010) Hydrogen protects vestibular hair cells from free radicals. Acta Otolaryngol Suppl 563:95-100

Tavazzani E, Spaiardi P, Zampini V et al (2016) Distinct roles of Eps8 in the maturation of cochlear and vestibular hair cells. Neuroscience 328:80-91 
Tian CJ, Kim YJ, Kim SW et al (2013) A combination of cilostazol and Ginkgo biloba extract protects against cisplatin-induced Cochleovestibular dysfunction by inhibiting the mitochondrial apoptotic and ERK pathways. Cell Death Dis. 4(2):e509

Tian C, Kim YJ, Lim HJ et al (2014) Red ginseng delays age-related hearing and vestibular dysfunction in C57BL/6 mice. Exp Gerontol 57:224-232

Varela-Nieto I, Morales-Garcia JA, Vigil P et al (2004) Trophic effects of insulin-like growth factor-I (IGF-I) in the inner ear. Hear Res 196(1-2):19-25

Wall C 3rd, Merfeld DM, Rauch SD, Black FO (2002) Vestibular prostheses: the engineering and biomedical issues. J Vestib Res 12(2-3):95-113

Wangemann P (2002) K+ cycling and the endocochlear potential. Hear Res 165(1-2):1-9

Watanabe K, Hess A, Bloch W, Michel O (2000) Expression of inducible nitric oxide synthase (iNOS/NOS II) in the vestibule of guinea pigs after the application of cisplatin. Anticancer Drugs 11(1):29-32

Watanabe K, Jinnouchi K, Baba S, Yagi T (2001) Induction of apoptotic pathway in the vestibule of cisplatin (CDDP)-treated guinea pigs. Anticancer Res 21(6a):3929-3932

Williams LH, Miller KA, Dahl HH, Manji SS (2013) Characterization of a novel ENU-generated myosin VI mutant mouse strain with congenital deafness and vestibular dysfunction. Hear Res 299:53-62

Wong EY, Xu CY, Brahmachary M, Xu PX (2016) A Novel ENUinduced mutation in myo6 causes vestibular dysfunction and deafness. PLoS ONE 11(5):e0154984

Wu X, Cai J, Li X et al (2017) Allicin protects against cisplatin-induced vestibular dysfunction by inhibiting the apoptotic pathway. Eur J Pharmacol 805:108-117

Xiang M, Gan L, Li D et al (1997) Essential role of POU-domain factor Brn-3c in auditory and vestibular hair cell development. Proc Natl Acad Sci U S a 94(17):9445-9450
Yamasoba T, Lin FR, Someya S et al (2013) Current concepts in agerelated hearing loss: epidemiology and mechanistic pathways. Hear Res 303:30-38

Yamoah EN, Lumpkin EA, Dumont RA et al (1998) Plasma membrane $\mathrm{Ca} 2+-A T P a s e$ extrudes $\mathrm{Ca} 2+$ from hair cell stereocilia. J Neurosci 18(2):610-624

Yoshida S, Sugahara K, Hashimoto M et al (2015) The minimum peptides of IGF-1 and substance P protect vestibular hair cells against neomycin ototoxicity. Acta Otolaryngol 135(5):411-415

Yousaf R, Gu C, Ahmed ZM et al (2018) Mutations in Diphosphoinositol-Pentakisphosphate Kinase PPIP5K2 are associated with hearing loss in human and mouse. PLoS Genet. 14(3):e1007297

Yu C, Rahmani M, Dent P, Grant S (2004) The hierarchical relationship between MAPK signaling and ROS generation in human leukemia cells undergoing apoptosis in response to the proteasome inhibitor Bortezomib. Exp Cell Res 295(2):555-566

Zhang M, Liu W, Ding D, Salvi R (2003) Pifithrin- $\alpha$ supresses p53 and protects cochlear and vestibular hair cells from cisplatin-induced apoptosis. Neuroscience 120(1):191-205

Zhang D, Fan Z, Han Y et al (2012) Apoptosis-inducing factor is involved in gentamicin-induced vestibular hair cell death. ORL J Otorhinolaryngol Relat Spec 74(1):1-5

Zheng JL, Helbig C, Gao WQ (1997) Induction of cell proliferation by fibroblast and insulin-like growth factors in pure rat inner ear epithelial cell cultures. J Neurosci 17(1):216-226

Publisher's Note Springer Nature remains neutral with regard to jurisdictional claims in published maps and institutional affiliations. 